\title{
Percepción de riesgo sobre lesiones no intencionales en niños y niñas, México
}

\author{
Elizabeth Sevilla, Mg. \\ Profesora Universidad de Guadalajara, \\ México*
}

\author{
Arturo Franco-Ordaz
}

Universidad de Guadalajara, México ***

rosbeth@gmail.com

\section{Resumen (analítico)}

Es posible evitar las lesiones no intencionales, particularmente en los menores, esto se ve obstruido por la percepción de los cuidadores. La narrativa recupera la experiencia de los cuidadores, padres, familiares, analizando la visión sobre las lesiones, en hijos o menores a cargo, hospitalizados. A partir de los resultados se percibe que hay una reducción de la percepción de riesgo en los cuidadores y carencia en la información de medidas preventivas. Además, una creencia de carencia de control, cedido al destino. Se concluye que existe una ingenuidad preventiva en la sobreestimación de las capacidades de los niños. Una percepción de riesgo, creencias y conductas que reducen la valoración del peligro. Roles de género y estatus socio-económico son bases de esta visión fatalista. Resultando la reducción de capacidades de prevención, hacia hijos o menores a cargo.

\section{Palabras clave}

Percepción, lesiones, accidente, niño, cuidadores, riesgo, rutina, hospitalización, prevención.

\section{Tesauro}

Biblioteca virtual en salud, Descriptores de Ciencias de la Salud, Bireme, Centro Latinoamericano y del Caribe de Información en Ciencias de la Salud.
Para citar este artículo

Sevilla, E., Ramos-Escobedo, A., \& Franco-Ordaz, A. (2019). Percepción de riesgo sobre lesiones no intencionales en niños y niñas, México. Revista Latinoamericana de Ciencias Sociales, Niñez y Juventud, 17(2), 1-22 doi:10.11600/1692715x.17208

Historial

Recibido: 18.07.2018

Aceptado: 22.11.2018

Publicado: 31.06.2019

Información artículo

Este artículo forma parte del proyecto denominado «Percepción de riesgo en el estudio de accidentes de niños menores de 5 años de edad: Un estudio de la zona metropolitana de Guadalajara, Jalisco México» registrado en la Dirección de Desarrollo en Salud, Departamento de Investigación, No. de registro 1244/13. Realizada entre mayo de 2014 y todavía en curso. Área: Sociología. Subárea: Temas especiales. 


\title{
The perception of risk of unintentional injuries among children in Mexico
}

\section{Abstract (analytical)}

It is possible to avoid unintentional injuries, particularly in children. This is affected by the perceptions of caregivers. This study collects the experiences of caregivers, parents and relatives, analyzing their visions of injuries of their children or children under their care who have been hospitalized. Based on the results it is perceived that there is a reduction of the perception of risk among caregivers and a lack of information on preventive measures. In addition, there is a belief that they have a lack of control and that it is unavoidable that children injure themselves. The authors conclude that there is a preventative naivety that exists among parents and caregivers and an overestimation of the abilities of children. There are perceptions of risk, beliefs and behaviors that reduce the levels of danger perceived by adults. Traditional gender roles and socio-economic status form the basis of this fatalistic vision. This results in a reduction of prevention capacities that protect adults' children and children under their care.

Keywords

Perception, injuries, accident, child, caregivers, risk, routine, hospitalization, prevention.

\section{Percepção de risco sobre lesões não intencionais em meninos e meninas no México}

\author{
Resumo (analítico)
}

É possível evitar lesões não intencionais, particularmente em crianças, ainda que obstruídas pela percepção dos cuidadores. A narrativa recupera a experiência dos pais ou familiares cuidadores, analisando a visão sobre as lesões, em crianças ou menores responsáveis, internados. A partir dos resultados nota-se que há redução na percepção do risco nos cuidadores e falta de informação sobre medidas cautelares. Além disso, uma crença de falta de controle, dada ao destino. Há também ingenuidade preventiva e superestimação das capacidades das crianças. Uma percepção de risco, crenças e comportamentos que reduzem a avaliação do perigo. Os papéis de gênero e o status socioeconômico são a base dessa visão fatalista. Redução resultante de capacidades de prevenção, para crianças ou menores responsáveis.

Palavras-chave

Percepção, lesões, acidente, criança, cuidadores, risco, rotina, hospitalização, prevenção.

\section{Información autores}

[*] Médica Cirujana y Partera. Maestra en Salud Pública por la Universidad de Guadalajara, Epidemióloga por el Instituto Mexicano del Seguro Social. Profesora Investigadora del Departamento de Ciencias Sociales del Centro Universitario de Ciencias de la Salud de la Universidad de Guadalajara. Orcid: 0000-0002-6935-5788. Índice H5: 3.5. Correo electrónico: rosbeth@gmail.com

[**] Psicólogo, Maestro en Antropología Social por el Colegio de Michoacán, Doctorado en Ciencias Sociales por la Universidad de Guadalajara. Profesor Investigador del Departamento de Ciencias Sociales del Centro Universitario de Ciencias de la Salud de la Universidad de Guadalajara. Orcid: 0000-0003-4453-4998. Índice H5: 0.7. Correo electrónico: thano45@hotmail.com

[***] Psicólogo por la Universidad de Guadalajara. Índice H5: 0. Correo electrónico: psicofranco93@gmail.com 


\section{Introducción}

En este estudio se explora y analiza la percepción de riesgo de cuidadores cuyos hijos e hijas fueron hospitalizados a consecuencia de alguna lesión no intencional sufrida. La primera causa de muerte, en menores de cinco años, son las lesiones no intencionales (Atak, Karaoglu, Korkmaz, \& Usubutun, 2010; Lourenço, Furtado, \& Bonfim, 2008). En cualquier tipo de lesión no intencional, se considera que es posible la prevención; no obstante, la percepción del riesgo que se tenga sobre estos puede llegar a modificar la conducta preventiva (Leeberk, Anuradha, Kuryan, \& Anuratha, 2017).

Mientras que en otros países se ha abordado el tema de la percepción del riesgo sobre accidentes en niños y niñas (Hogan, Weaver, Cioni, Hamilton, \& Thompson, 2018; Whitehead \& Owens, 2012), en Latinoamérica se identificó la percepción del riesgo en mujeres embarazadas sobre accidentes en niños (Cassus-Becerra, Fuentes-Vásquez, \& Valdés-Valenzuela, 1998), las acciones de cuidado y prevención (Chardon, 2008) con profesionales de enfermería y su percepción del riesgo con objetos cortopunzantes (Marques, Bautista, Teixeira, Fortes, \& Sales, 2012), o que la falta de conocimiento sobre la susceptibilidad de sufrir accidentes los favorece (Gurgel \& Monteiro, 2016). Por su parte, en México es todavía escasa la información publicada; la mayoría de los estudios realizados tienen un enfoque cuantitativo en el que, pese a su aporte e importancia, por lo común en su explicación no se toman en cuenta factores socioculturales interrelacionados, como la perspectiva de los cuidadores.

Así, surge la siguiente pregunta de investigación: ¿cuáles factores culturales intervienen en la disminución de la percepción de riesgo de los cuidadores en la incidencia de lesiones no intencionales de niños y niñas, y cómo lo hacen? Para dicho propósito se plantea explorar y analizar la experiencia de los cuidadores acerca de las lesiones no intencionales, por medio de la narrativa como estrategia antropológica y cualitativa. El objetivo del estudio es explorar la percepción del riesgo de los cuidadores y su relación con las lesio- 
nes no intencionales de niños y niñas que, como consecuencia de ello, tuvieron que ser hospitalizados.

Algunas condiciones sociales estructurales de la familia, tales como sector social (García \& De Oliveira, 1998), espacios reducidos, economía precaria, bajo nivel educativo, madre trabajadora, padre ausente, favorecen la presencia de lesiones. Igualmente, intervienen aspectos culturales, como hábitos y costumbres que participan de manera importante en la percepción del riesgo, así como en la explicación del evento por parte de los cuidadores con ideas de fatalismo o creencias místico-religiosas. Los adultos suponen que los menores entienden que cosas son riesgosas, cuando en realidad el niño o la niña solo «aprende» — condicionamientos- a alejarse de objetos o espacios prohibidos, como consecuencia de llamadas de atención o, en ocasiones, de castigos físicos. En realidad, el niño y la niña aún no comprenden cognitivamente el riesgo o peligro, y no poseen una percepción de riesgo; su natural inquietud, instinto y necesidad de exploración, los llevan a ponerse frecuentemente en calidad de víctima potencial de lesiones. Los niños y las niñas no comprenden qué comportamientos son peligrosos hasta que cognitivamente pueden entender la relación entre causa y efecto.

Los adultos, en cambio, juzgan el riesgo de acuerdo con sus procesos cognitivos y la comprensión que hagan de los fenómenos, y, con base en ello, toman decisiones (Oltedal, Moen, Klempe, \& Rundmo, 2004; Slovic, Fischhoff, \& Lichtenstein, 2011). Por esto, cuando la probabilidad de disminuir un riesgo demuestra mayor efectividad, con un margen de error muy bajo, implementan acciones, con la certeza de que el costo y el tiempo de inversión se han compensado (Slovic et al., 2011). De hecho, cuando se percibe un riesgo ante un evento que se identifica como grave, el efecto que produce se traduce en un cambio de comportamiento para impedir que suceda (Ferrer \& Klein, 2015; Vasvàri, 2015). Realizar diferentes rutinas hogareñas a lo largo de semanas y meses brinda cierta utilidad, pero termina por reducir o anular el riesgo real existente en el hogar en la percepción de los adultos.

\section{Perspectiva}

La experiencia del accidente es una acción simbólica en los padres de los menores lesionados. La perspectiva utilizada en este estudio es el constructivismo social, acercamiento que representa la racionalidad hermenéutico-interpretativa. Se trata de la experiencia de los padres o familiares de los niños lesionados y hospitalizados. Como señala 
Schutz, de lo que se trata es de establecer un estudio «objetivo» de los significados «subjetivos» (Schutz, 1974), es decir, de los actores. Se recurre a tradiciones teóricas que comparten el principio de que el comportamiento humano involucra acción simbólica, y que la experiencia comprende subjetividad, interpretación y significados. La primera corresponde a la tradición antropológica de Geertz (1988), puesto que el conjunto de lesiones sufridas es un producto cultural susceptible de ser interpretado. En este orden de ideas, el concepto de significado está relacionado con los términos que describen la experiencia e interpretación acerca del accidente de los hijos de los actores, por lo que el significado se refiere a las fórmulas que los actores usan para definir lo que les sucede; las interpretaciones que hacen de su experiencia (Geertz, 1988). La segunda corresponde también a la antropología, ya que la obtención de la experiencia del accidente y la aproximación a la percepción de riesgo se hacen por medio de la narrativa, esto es, como experiencia antropológica (Bruner, 1986; Turner, 1986).

En cuanto al término accidente, este se asocia a un hecho azaroso, que se presenta de manera imprevisible (Davis \& Pless, 2001; Zayas-Mujica, Cabrera-Cárdenas, \& Cayón, 2007), concepción que elimina toda posibilidad de contextualizarlo como prevenible. Por tal motivo, en el presente documento se privilegia conceptualmente el vocablo lesión: «Menoscabo orgánico que resulta de la exposición a una energía mecánica, térmica, eléctrica, química o radiante, que interactúa con el organismo en cantidades o con un índice que excede el límite de la tolerancia fisiológica» (Organización Panamericana de la Salud, 2004, p. 9), por lo que lesión no intencional alude a «conjunto de circunstancias y factores sociales, educativos, ambientales, económicos, culturales, políticos, urbanísticos y jurídicos» (Organización Panamericana de la Salud, 2004, p. 9), ya que además existen lesiones que son resultado de maltrato infantil - violencia- y que en ningún sentido se deben considerar como accidentes.

Se define riesgo como «la probabilidad de que un evento ocurra» (Tocabens, 2011, p. 2), en tanto que percepción de riesgo es un «fenómeno complejo que puede ser determinado por factores sociales, psicológicos, físicos, políticos o culturales» (Kouabenan, 2009, p. 770), por lo que puede ser muy diferente en relación con la estimación del riesgo objetivo o real. Este no depende de las percepciones de la persona o del conocimiento que pueda tener acerca del riesgo y de las preocupaciones del riesgo. La definición del concepto de riesgo que la persona tenga influye en la forma como acepta los riesgos en la vida y la actitud frente a este (Slovic, 2012; Whitehead \& Owens, 2012). Aquí se asume la 
importancia del concepto de percepción del riesgo para explicar las lesiones acontecidas en el grupo estudiado.

\section{Metodología}

Se hizo una búsqueda de información en varias bases de datos: Pubmed, ScienceDirect, Scopus, BVS, ProQuest, Ebsco, con palabras claves: risk perception, injury, children.

\section{Tiempo, lugar y persona de las entrevistas}

Inicialmente, se consideró incluir solo a los cuidadores de menores de cinco años, pero al final se incluyó a todos los niños y niñas que se encontraron hospitalizados por alguna lesión no intencional para tener un margen más amplio de casos. De esta manera, se encontraron niños en edades que van desde los seis meses hasta los nueve años.

Los sujetos de estudio eran pacientes del Nuevo Hospital Civil Juan I. Menchaca, de segundo nivel de atención médica, localizado en la ciudad de Guadalajara (Jalisco), en el que se atiende a población de escasos recursos económicos. Se solicitó consentimiento informado y autorización para grabar la entrevista; se empleó la entrevista cara a cara, semiestructurada y a profundidad. La entrevista semiestructurada es aquella que está basada en una guía de entrevista (Bernard, 1988). Se utilizó una muestra teórica y, por lo tanto, una estrategia cualitativa y un análisis de la información.

\section{Tipo de investigación}

La naturaleza de la pregunta implicó la utilización de una muestra teórica y una estrategia cualitativa en la recolección y análisis de la información para mostrar la experiencia de los cuidadores en la lesión.

\section{Participantes}

Se entrevistó a los cuidadores de niños y niñas hospitalizados por alguna lesión no intencional para conocer su percepción del evento y por asumir que estos son quienes tienen la responsabilidad de cuidar a los menores, además de que fueron los que estuvieron presentes al momento de acudir a realizar la entrevista. Se recuperó la experiencia por medio de un planteamiento hermenéutico que se centró en el relato de la experien- 
cia desde el punto de vista del cuidado, y de cómo se concibió el evento a través de sus causales y de las consecuencias que este trajo.

\section{Calidad de la información}

Se consideraron tres criterios para asegurar la calidad de la información:

- Que la persona entrevistada suministrara la información en cantidad suficiente para elaborar una historia de caso.

- Que la información tuviera relación y un grado significativo de veracidad con respecto a los referentes clínicos y médicos.

- Que la información se proporcionara con completa libertad de decisión del tutor/padres/cuidador, o de quien se encontrara al cuidado del niño o la niña al momento de la entrevista en el hospital.

\section{Manejo de la información}

Para lograr uniformidad y facilitar el proceso de análisis, la transcripción se efectuó siguiendo el formato de las normas de transcripción de Robert Weber (1990). Párrafos de las entrevistas fueron la unidad de análisis.

Se utilizó el análisis temático (De Souza Minayo, 1995) para descubrir los núcleos de sentido que componen una comunicación cuya presencia o frecuencia signifiquen alguna cosa para el objetivo trazado. El análisis temático se encaminó cualitativamente a la presencia de determinados temas, denotando los valores de referencia y los modelos de comportamiento que están en el discurso (De Souza Minayo, 1995).

\section{Resultados}

\section{Factores en torno a las lesiones}

\section{¿Qué es un accidente?}

Los accidentes en niños y niñas no solo están relacionados con diversos tipos de traumatismos, heridas o quemaduras, sino que también están rodeados de aspectos como el cuidado, responsabilidad o culpa; asimismo, hay una explicación acerca de su ocurren- 
cia -el azar, el infortunio, la fatalidad y el riesgo-, por lo que llegan a ser considerados en ocasiones como parte inherente de su vida y como eventos ante los que nada se puede hacer para evitar que sucedan: «Yo pienso que no se pudo haber evitado... Fue algo rápido, no se pudo haber evitado» (participante og; en adelante solo se referenciará su número).

Algunos padres llegan a admitir plenamente su desatención como el detonante para la aparición del accidente, incluso deslindando de toda responsabilidad a los niños y niñas, considerando que estos todavía no identifican los riesgos ni razonan sobre cómo cuidarse: «Es un accidente, para mí lo que le pasó a él es un descuido de nosotros; ¿por qué? Porque él no tiene razón» (o4).

Otros cuidadores fueron más categóricos al señalar que de haber atención y cuidado de los padres, los accidentes «no existirían»: «No creo que haya accidentes eda (sic); bueno, sí hay accidentes, y como suelen pasar, pero pues si te fijas yo creo que no» (o8).

Aún dentro de una particular idiosincrasia, este último aspecto es el que debe permear en la percepción del riesgo para advertir lesiones, esto es, debe existir una convicción tal acerca de la responsabilidad del cuidador.

\section{El riesgo}

En la tabla 1 aparece información que permite acercarse a la reconstrucción de hechos que llevaron a vivir la lesión y, a la vez, visibilizar la situación del menor y los cuidadores, ya sean sus padres, parientes o tutores. Estos se encuentran dentro de un contexto que los lleva a realizar múltiples actividades dentro del hogar o fuera de este, al mismo tiempo que tienen la responsabilidad del cuidado del menor.

\section{La incomunicación de los riesgos}

Es necesario aclarar que muchos de los riesgos se acrecientan o disminuyen dependiendo de las edades, de las circunstancias físicas habitacionales, de las condiciones económicas, al igual que de las actitudes y mentalidades en relación con aspectos de cuidado y prevención. Además, se suma otro factor relevante: la falta de comunicación sobre la presencia de estos, así como características de los sistemas de cuidado familiar: «como mi suegra nunca, mi suegro no sabía ni qué era... Entonces, este... Primero como que informar sabes qué ira, esta es soda, va a estar aquí, atrás del este y la fregada (sic) para que no lo agarren» (10). 


\section{Tabla 1}

Características y condiciones de las lesiones no intencionales en niños

\begin{tabular}{|c|c|c|c|c|c|c|c|c|c|c|c|c|c|}
\hline Entrevista & Edad & Género & Tiempo & Estado Civil & Ocupación & Escolaridad & $\begin{array}{l}\text { Número } \\
\text { de Hijos }\end{array}$ & $\begin{array}{c}\text { Habitantes en } \\
\text { Vivivienda }\end{array}$ & $\begin{array}{c}\text { Presencia de } \\
\text { Adulto en } \\
\text { Evento }\end{array}$ & $\begin{array}{c}\text { Actividad del } \\
\text { Menor en el } \\
\text { Momento }\end{array}$ & $\begin{array}{c}\text { Actividad del Adulto } \\
\text { en el Momento }\end{array}$ & $\begin{array}{c}\text { Menor } \\
\text { Solo }\end{array}$ & Entrevistada/0 \\
\hline $\begin{array}{c}\text { Caida de } \\
\text { Azotea-01 }\end{array}$ & $\begin{array}{c}3 \\
\text { años }\end{array}$ & Femenino & $14: 00$ & Separada & $\begin{array}{l}\text { Actividad } \\
\text { Laboral } \\
\text { Presente }\end{array}$ & $x$ & 4 & 6 & Sí & $\begin{array}{c}\text { Ayudando a } \\
\text { la madre } \\
\text { (Tender Ropa) }\end{array}$ & Tender Ropa & No & Madre \\
\hline $\begin{array}{l}\text { Quemadura } \\
\text { con Té-02 }\end{array}$ & $\begin{array}{c}2 \text { años } \\
7 \\
\text { Meses }\end{array}$ & Masculino & $\begin{array}{l}21: 00- \\
21: 30\end{array}$ & Casada & $x$ & $x$ & 3 & 5 & Sí & $\begin{array}{c}\text { Paso por la } \\
\text { estufa }\end{array}$ & $\begin{array}{c}\text { Madre salio a la } \\
\text { tienda - Padre en el } \\
\text { lugar }\end{array}$ & No & Madre \\
\hline $\begin{array}{l}\text { Quemadura } \\
\text { con Birria - } 03\end{array}$ & 3 Años & Masculino & $\begin{array}{c}\text { Tarde } \\
\text { (Hora de } \\
\text { Comida) }\end{array}$ & $x$ & $x$ & $x$ & $x$ & $x$ & Sí & $\begin{array}{c}\text { Esperando a } \\
\text { hacer } \\
\text { compras con } \\
\text { el padre }\end{array}$ & $\begin{array}{c}\text { Distraido ayudando } \\
\text { a un vendedor }\end{array}$ & No & Padre y Madre \\
\hline $\begin{array}{c}\text { Ingesta Sosa } \\
\text { (Atomizador) } \\
\text { Esposo-04 } \\
\end{array}$ & $\begin{array}{l}1 \text { año } \\
1 \text { mes }\end{array}$ & Masculino & 08:00 & Union Libre & Trailers & Primaria & 1 & 3 & No & $\begin{array}{l}\text { Jugando solo } \\
\text { en un pasillo }\end{array}$ & Bañandose & Sí & Padre \\
\hline $\begin{array}{c}\text { Ingesta Sosa } \\
\text { (Atomizador) } \\
\text { Esposa-05 }\end{array}$ & $\begin{array}{l}1 \text { año } \\
1 \text { mes }\end{array}$ & Masculino & 09:00 & Union Libre & Desempleada & $x$ & 1 & 3 & No & $\begin{array}{l}\text { Jugando solo } \\
\text { en un pasillo }\end{array}$ & Peinandose & Sí & Madre \\
\hline $\begin{array}{c}\text { Ingesta de } \\
\text { Sosa Caustica } \\
06 \\
\end{array}$ & $\begin{array}{c}1 \text { año } \\
8 \\
\text { meses }\end{array}$ & Masculino & 12:00 & Union Libre & Comerciante & Secundaria & 3 & $x$ & Sí & $\begin{array}{l}\text { Junto a la } \\
\text { madre }\end{array}$ & $\begin{array}{l}\text { Lavando ropa (junto } \\
\text { al niño) }\end{array}$ & No & Madre \\
\hline $\begin{array}{c}\text { Caida de } \\
\text { Escalera-07 }\end{array}$ & $\begin{array}{c}1 \text { año } \\
8 \\
\text { meses }\end{array}$ & Femenino & $\begin{array}{c}\text { Tarde } \\
\text { (Viendo la } \\
\text { tele) }\end{array}$ & Unión Libre & $\begin{array}{l}\text { Empleada } \\
\text { Domestica }\end{array}$ & Primaria & 3 & 5 & No & $\begin{array}{l}\text { Acababa de } \\
\text { despertar y } \\
\text { subio las } \\
\text { escaleras }\end{array}$ & $\begin{array}{c}\text { Preparar leche en la } \\
\text { cocina }\end{array}$ & Sí & Madre \\
\hline $\begin{array}{l}\text { Golpe Contra } \\
\text { Ladrillos- } 08\end{array}$ & $\begin{array}{l}1 \text { año } \\
8 \text { días }\end{array}$ & Masculino & $\begin{array}{c}\text { Se } \\
\text { descooce }\end{array}$ & Soltera & $\begin{array}{l}\text { Empleada } \\
\text { juegos } \\
\text { mecanicos }\end{array}$ & Primaria & 4 & 8 & $x$ & $x$ & $x$ & $x$ & Madre \\
\hline $\begin{array}{c}\text { Caida } \\
\text { escaleras y } \\
\text { golpe al subir } \\
\text { al auto - } 09\end{array}$ & 9 años & Masculino & 19:00 & Casados & Comerciantes & Secundaria & 5 & 7 & Sí & $\begin{array}{c}\text { Bajando las } \\
\text { aescaleras y } \\
\text { subiendo al } \\
\text { auto, para } \\
\text { salir an }\end{array}$ & $\begin{array}{c}\text { Padres presentes, le } \\
\text { esperaban dentro de } \\
\text { vehiculo }\end{array}$ & No & Abuela \\
\hline $\begin{array}{c}\text { Ingestión de } \\
\text { Sosa Caustica } \\
2-10\end{array}$ & $\begin{array}{c}3 \text { años } \\
4 \\
\text { meses }\end{array}$ & Masculino & $17: 30$ & Casados & $\begin{array}{c}\text { Empleado en } \\
\text { Transito }\end{array}$ & Preparatoria & 1 & 3 & No & $\begin{array}{c}\text { Jugando a } \\
\text { "las } \\
\text { escondidas" }\end{array}$ & $\begin{array}{c}\text { Jugar escondidas } \\
\text { con el (abuelo). } \\
\text { Salir a correr } \\
\text { (padre) }\end{array}$ & Sí & Padre \\
\hline $\begin{array}{c}\text { Caída de } \\
\text { Cama-11 } \\
\end{array}$ & $\begin{array}{c}6 \\
\text { Meses }\end{array}$ & Masculino & $\begin{array}{l}\text { 10:00 } \\
11: 00\end{array}$ & Union Libre & Hogar & Preparatoria & 1 & 3 & No & $\begin{array}{c}\text { Acababa de } \\
\text { despertar }\end{array}$ & En la cocina & Sí & Madre \\
\hline $\begin{array}{c}\text { Caída de } \\
\text { Carreola-12 }\end{array}$ & $\begin{array}{c}1 \text { mes } \\
15 \\
\text { días }\end{array}$ & Masculino & $16: 00$ & Union Libre & Hogar & Secundaria & 3 & 9 & No & $\begin{array}{l}\text { Se encontraba } \\
\text { en la carreola }\end{array}$ & $\begin{array}{c}\text { Se alejaba de la } \\
\text { carreola }\end{array}$ & Sí & Madre y Abuela \\
\hline $\begin{array}{c}\text { Caida de } \\
\text { Escaleras 2- } \\
13 \\
\end{array}$ & $\begin{array}{c}3 \text { años } \\
10 \\
\text { Meses }\end{array}$ & Masculino & $18: 00$ & Soltera & Hogar & Preparatoria & 2 & 6 & Sí & $\begin{array}{c}\text { Recogía } \\
\text { zapatos en } \\
\text { las escaleras }\end{array}$ & $\begin{array}{c}\text { Junto a el subiendo } \\
\text { escaleras }\end{array}$ & No & Madre \\
\hline $\begin{array}{c}\text { Ingesta de } \\
\text { Acetona - } 14\end{array}$ & 2 Años & Femenino & $x$ & Casada & $x$ & $x$ & 2 & 4 & Sí & $\begin{array}{c}\text { Jugaba con el } \\
\text { frasco de } \\
\text { acetona }\end{array}$ & $\begin{array}{c}\text { Peinandose, frente a } \\
\text { ellas }\end{array}$ & No & Madre \\
\hline $\begin{array}{c}\text { Ingesta de } \\
\text { Sosa Caustica } \\
3-15 \\
\end{array}$ & 5 Años & Masculino & $15: 00$ & Casada & $x$ & $x$ & 2 & 5 & No & SD & Fue a la cocina & Sí & Madre \\
\hline $\begin{array}{c}\text { Quemadura } \\
\text { en la cocina - } \\
16\end{array}$ & 4 Años & Femenino & $16: 00$ & Casada & $x$ & $x$ & 2 & 6 & Sí & $\begin{array}{c}\text { Ayudar en la } \\
\text { cocina (se } \\
\text { subio a la } \\
\text { estufa) }\end{array}$ & Cocinando & No & Abuela \\
\hline $\begin{array}{l}\text { Golpe con } \\
\text { Rodilla -17 }\end{array}$ & $\begin{array}{l}3 \text { Años } \\
1 \text { Mes }\end{array}$ & Femenino & Noche & Casada & Hogar & Secundaria & 3 & 5 & Sí & $\begin{array}{c}\text { En la cama } \\
\text { (reñia con su } \\
\text { hermano) }\end{array}$ & En el baño & No & Madre \\
\hline $\begin{array}{c}\text { Caída } \\
\text { Jugando a la } \\
\text { Cuerda - } 18\end{array}$ & 7 Años & Masculino & 13:30 & Union Libre & Hogar & Secundaria & 3 & 13 & Sí & $\begin{array}{c}\text { Jugando a "la } \\
\text { cuerda" }\end{array}$ & En su habitación & No & Madre \\
\hline
\end{tabular}


Los relatos reflejan que algunas lesiones habrían podido evitarse si hubiera existido cierta información con otros miembros de la familia sobre cómo utilizar determinados enseres domésticos de riesgo.

\section{Prevenir el riesgo}

Las madres admiten que cuando el niño o niña no está a la vista y bajo su atención, su vida se convierte en un mundo de completa incertidumbre, con múltiples circunstancias de inseguridad, las mismas que son difíciles de controlar. En el sector social popular, la necesidad de que la madre trabaje como asalariada o en labores extradomésticas (García \& De Oliveira, 1998) tiene relevancia, ya que en ocasiones las madres reconocen conscientemente un mundo arriesgado para los pequeños como parte de sus circunstancias generales de vida: «Sí, todo es riesgo... A veces tú estás muy ocupada porque ya te vas a ir a trabajar y cuando menos esperas ya el niño está tomando eso, o... o ya se cayó de las escaleras (...) o se salió a la calle y lo aventó un carro; o sea, es que tú no sabes realmente... cuándo va a pasar un accidente así (o6).

Entre las soluciones dadas por los cuidadores, realmente sorprende el grado de ingenuidad - preventiva - expresada, en aras de conseguir una ventaja cotidiana. En su inocencia, el cuidador cree que los pequeños se detendrán ante una etiqueta o «estampita», lo que puede indicar ciertas formas de pensar, detrás de lo cual se encuentran ideas equivocadas y la generación de lesiones: «tenga una estampita de peligro y que uno lo ponga así a la mano, sabiendo que un niño ya sabe, y pues uno ya sabe que es un riesgo para él, y no lo quite, que ahí lo deje» (o4).

Por difícil que parezca, estos son los detalles que terminan conformando actitudes de predisposición en los cuidadores ante la incidencia de lesiones.

\section{Vida cotidiana: «el enemigo en casa»}

Los tipos de lesiones que presentaron los niños y niñas sucedieron dentro del contexto de actividades de la vida cotidiana. Debido a la prioridad otorgada a las actividades hogareñas y rutinas acostumbradas por parte de los cuidadores, la percepción de riesgo quedó reducida o anulada.

Los relatos señalan que, en forma frecuente, las madres se hacen acompañar de los pequeños para «estar al pendientes» de su seguridad o por simple apego del menor, pero este acompañamiento en ocasiones resulta desacertado si el cuidador no toma en cuenta 
otras condiciones contextuales. La atención de la madre no está del todo puesta en el menor, sino dividida en la actividad que realiza. Dicha atención fragmentada conlleva riesgos: «... pues yo creo [que] fue cuando se me fue [cayó de segundo piso], porque yo también por estar tendiendo la verdad también no... no me fijé» (o1).

\section{Medidas pseudopreventivas}

Un aspecto crucial en las lesiones de pequeños son las medidas «preventivas» que los familiares adoptan y que, desde su perspectiva, atienden el riesgo. Pese a ello, algunas de las medidas utilizadas resultan erróneas y no eliminan la probabilidad de que se suscite un daño: «la metí hasta adentro [soda cáustica] y ahí no la alcanzaba, porque no camina, no alcanza mucho la mano, y si la alcanza, si se llega a parar y la jala, si la ha de ver» (05).

En los testimonios anteriores existe una clara subestimación de las capacidades de los pequeños, esto es, la medida preventiva se realiza en función de lo que se cree que no pueden efectuar: «no camina», «no alcanza», «no se puede subir».

Guardar materiales peligrosos para los infantes es una práctica común en ciertos grupos de la población, que obedece a un sentido de economía familiar. Este círculo vicioso (en el que los padres son un eslabón) tiene su inicio en el proceso de venta inadecuada por parte de los comercios, los cuales expenden el producto en envases de alimento. Además, a pesar de ser productos para uso industrial, como la soda cáustica, las madres de familia lo utilizan de manera indebida en el hogar por sus propiedades de limpieza.

La expresión «nunca pensé», dicha inmediatamente después de la lesión, implica que los familiares no pensaron que el hecho de guardar la sustancia peligrosa en un frasco de alimento fuera algo inadecuado; de igual manera, implica sorpresa que el niño fuera capaz de consumir algo que no debía y, finalmente, el desconocimiento acerca de que los niños y niñas son curiosos por naturaleza: «Nunca pensé, la verdad, que fuera a tomar esa cosa; como venía en galón de leche...» (o6).

\section{La utilidad del riesgo frente al costo de la prevención}

¿Por qué los adultos aceptan circunstancias de riesgo para los niños en casa? Se pueden identificar por lo menos cuatro razones: la utilidad práctica cotidiana, un supuesto beneficio o ahorro económico, un sentido de confianza y control generado a través del tiempo sin que sucedan lesiones y, tal vez la más importante, que el cuidador también es 
rebasado por el tiempo, las premuras, la improvisación, las presiones y prioridades de orden económico, así como por su escaso conocimiento e información sobre aspectos preventivos, hábitos y costumbres. En otras palabras, la percepción del riesgo y la capacidad preventiva del cuidador quedan muchas veces limitadas y están influenciadas por los factores mencionados con anterioridad.

Lamentablemente, el adulto desestima el riesgo debido a la utilidad y el beneficio práctico obtenido en actividades hogareñas diversas, a pesar del grado de conciencia existente sobre la presencia en casa de sustancias o circunstancias físico-espaciales riesgosas. Aunque parezca sorprendente, en estos grupos de la población los riesgos son tolerados en parte por los beneficios prácticos y económicos que se obtienen.

\section{El peligro como costumbre}

La reducción del riesgo es una constante en la mayoría de las lesiones. Pese a que se puede observar de manera real la probabilidad del daño, no se tiene en cuenta, o bien se utilizan medidas inadecuadas para resolverlo. Debido a las precarias condiciones generales de vida y en ocasiones a las profundas necesidades de supervivencia, el riesgo no se niega, pero sí se desestima y desatiende.

Otro aspecto importante para la existencia de lesiones es el tiempo de exposición. Cuando durante largo tiempo se ha mantenido en el hogar una actividad riesgosa sin que ocurra un daño, suele generarse una falsa percepción de control y seguridad en el individuo. Parece que no fuera necesario modificar nada, ya que así han funcionado las cosas durante todo el tiempo. Sin embargo, el riesgo no ha dejado de existir, solo que se ha dejado de percibir: «Pues serán cinco meses... seis (05) [que permanecía la botella con desengrasante en la casa]».

\section{Rutina y percepción de riesgo}

Algunas de las rutinas en el hogar hacen que el riesgo persista y sea latente la posibilidad de las lesiones; algunas veces son solo disfrazadas y ajustadas pensando en los niños y niñas de casa, pero no en un sistema de seguridad general que trascienda el propio hogar. Lo anterior puede observarse más comúnmente en tres circunstancias.

La primera de estas es aquella en que la medida preventiva adoptada le da al adulto una sensación de cierta seguridad a lo largo del tiempo; pero, a pesar de esto, factores 
imprevistos potencian el riesgo. En este caso particular, la madre esconde el recipiente con soda cáustica y la medida preventiva resulta relativamente efectiva, de acuerdo con las características de su hijo; sin embargo, un niño amigo de su hijo, que estaba de visita, saca la sustancia del escondite y se produce la lesión:

Es que yo la metí [envase con soda] bien metida hasta dentro, porque ahí no la alcanzaba, porque yo he dejado varias veces al niño ahí, y no, nunca lo ha alcanzado, y este, ahora pues yo creo el niño la bajó, la ha de haber acercado mucho a la orilla y el niño se ha de ver agarrado y se le vino encima la botellita (05).

El caso permite reafirmar que el adulto suele tomar medidas relativas de prevención, o sea, medidas que a la postre resultan considerablemente riesgosas para los niños. En los relatos hay indicios de que las rutinas cotidianas afectan la percepción del riesgo en el adulto. La repetición de ciertas actividades termina en rutina, por lo que se desatiende el riesgo debido a que se dejan de implementar las medidas adecuadas, que no reciben consecuencias negativas inmediatas (Marques et al., 2012).

La segunda de las circunstancias es alterar las actividades diarias, lo cual genera una lesión. Con la confianza de que no sucederá nada, la cadena de hábitos no se realiza al igual que siempre. Entre las rutinas del hogar, hacer lo acostumbrado suele incluir algunas medidas de prevención para los niños, y aunque estas sean ordinarias y mecánicas, otorgan cierto grado de previsión. No obstante, dejar de hacer lo habitual puede incrementar el riesgo y exponer al menor a sufrir lesiones. El siguiente testimonio muestra que la madre, al hacer cambios en el manejo acostumbrado de la soda cáustica, favoreció que sucediera la lesión: «en cuanto me tardé yo creo un minuto o dos y volteé... Sí, la verdad nunca hago eso, siempre llego del tianguis, este, por lo regular siempre me lo dan en bolsita y yo lo relleno en mis botes, pero nunca al alcance de los niños» (15).

El corto tiempo en el que suceden las lesiones está vinculado con la rutina en la creencia de ilusión de invulnerabilidad (Safiétou \& Kouabenan, 2013), en la que se aminora la capacidad de que un riesgo cause un daño al suspender la rutina de cuidado por segundos.

Dentro del tercer tipo de circunstancia, las rutinas también pueden corresponder a determinados comportamientos de los niños. Si bien dichos comportamientos infantiles contienen cierto factor de riesgo, el adulto los consiente, sin llegar a prever que estos pueden evolucionar y transformarse en serias lesiones: 
esa vez de la andadera pos (sic) salió rodando, por eso lo llevé, pero sí se le hizo «esa vez, hasta vomitó, le salió hasta sangre de la nariz, de la boca se me abrió, y luego aun así ni siquiera una radiografía, «ay, no nomás es el golpe, póngale, póngale hielo». «Ah, bueno», y yo me imagino que de esa vez porque de otra veces no se me golpea tan fuerte, y esa vez sí se me golpeó fuertísimo (o8).

Estos testimonios ponen de manifiesto que la presencia de las lesiones estriba en la ausencia de suspicacia del adulto, en la falta de una actitud de aprehensión sobre las posibilidades de ocurrencia de incidentes perjudiciales. Esto es lo que significaría en todo caso prever, es decir, anticiparse a la ocurrencia de hechos indeseables. Por desgracia, ser suspicaz o previsor en el cuidado de menores llega a interpretarse incluso como medidas exageradas por parte de los cuidadores. Por otro lado, deberían fomentarse el acceso a la información y la ejecución de campañas públicas que indiquen la peligrosidad de las andaderas.

\section{El cuidador}

\section{El rol del cuidador: su presencia no es sinónimo de cuidado}

La experiencia de las lesiones no intencionales señala que el cuidado de los pequeños requiere una atención constante y de carácter estricto por parte del adulto. En ocasiones, la simple presencia del adulto no es suficiente, ya que este puede confundir o desatender el factor de riesgo: «el niño estaba conmigo [en el lavadero], pero nunca, nunca... imaginé que se le iba a ser fácil el tomarle ahí a eso, yo pienso que se ha de haber figurado que era jugo o no sé» (o6).

En el relato del cuidador se advierten el reconocimiento y la presencia de factores de riesgo de gran importancia. No obstante su peligrosidad, en forma increíble el riesgo llega a tolerarse con base en una inexperiencia del daño. El término inexperiencia del daño se refiere a la actitud caracterizada por un sentimiento de seguridad, exceso de confianza, despreocupación o indiferencia ante determinados peligros por parte del cuidador, como resultado de la ausencia en el pasado de lesiones de consideración en el niño: «las escaleras... no tienen protección tampoco, o sea, están libres, y nunca, nunca nos había pasado esto, la verdad»(o7). 
El examen del conjunto de testimonios muestra que existió una presencia-ausencia del cuidador. Un aspecto que no puede dejar de mencionarse es que el cuidador adopta una actitud de jugar con el riesgo; es decir, basado en que el pequeño ya ha realizado antes un buen número de veces ciertas conductas — no desprovistas de riesgo- sin consecuencias negativas, se le continúa permitiendo practicarlas con base en un aprendizaje mal entendido: «Pues igual que se fuera por la pared. Es que normalmente así se sube, pero ese día iba a agarrar sus zapatos; entonces por eso, como los zapatos estaban en la pura orilla, por quererlos agarrar...» (13).

\section{Descuidos}

Sería interminable la serie de peligros y lesiones no intencionales que la presencia del cuidador previene y evita cotidianamente con tan solo alcanzar y agarrar a la criatura. No obstante, lo que hay que subrayar es que para la aparición de algunas lesiones solo se requieren segundos, a pesar de la presencia del cuidador: «la verdad, ya nomás cuando oí como que quiso detenerse con el lazo, y ya nomás cuando yo quise agarrar pues ya no pude, ya era demasiado tarde, se me fue pa bajo» (o1).

Existe una marcada diferencia entre el tiempo del evento (segundos) y el tiempo de riesgo o exposición continua al riesgo (horas, días, meses). Pese a que las lesiones suceden en segundos, el tiempo para que sobrevengan cuenta desde la exposición al riesgo hasta la presentación efectiva del daño.

Desde la percepción del cuidador, los segundos son una forma de medir el tiempo en el que tiene lugar el desenlace, pero también es una manera de interpretar su causalidad. Esto se complica cuando el tiempo y la obligación de cuidado recaen frecuentemente sobre las mujeres.

\section{Delegar el cuidado}

Las redes de apoyo familiar son la base de la presencia de determinados sistemas de cuidado de los pequeños. En nuestra cultura, es común que se delegue el cuidado de los pequeños a familiares en el hogar de estos últimos. En ocasiones, en dichos hogares hay riesgos desconocidos que pueden ser descubiertos por la inocencia infantil: «entonces este, que estaban jugando a las escondidas... y resulta que mi suegra... este, tenía la soda atrás de la estufa» (10). 


\section{Primeros auxilios, una oportunidad perdida}

Los relatos muestran que los primeros auxilios brindados por los familiares no fueron los indicados y en algunos casos fueron contraproducentes. En los casos de ingesta de soda cáustica está contraindicado provocar el vómito, ya que ocasiona una doble quemadura por el paso nuevamente del líquido por el esófago; sin embargo, esta constituyó la primera medida tomada: «yo ya al niño le lavé y todo e hice que vomitara» (10).

\section{La actitud fatalista}

Los accidentes tienen un antes y un después en cuanto a su interpretación y significado. El fatalismo, sobre todo, comprende creencias e ideas que otorgan explicación y aceptación de ciertos hechos dañinos una vez que estos han ocurrido. Constituye una forma de explicarse y sobrellevar experiencias difíciles en la vida, como las lesiones no intencionales (Foster, 1988).

El análisis de los relatos arroja la identificación de tres categorías que corresponden al fatalismo: fuera del control del individuo, el destino y la divinidad. A través de estas concepciones los cuidadores representan conformidad y aceptan la predeterminación del destino, adoptan una actitud resignada, en la que no se pueden predecir, decidir o guiar los hechos. Visto como una forma de adaptación del sujeto, se define como un fatalismo individualista (Blanco \& Díaz, 2007).

Es un hecho que las creencias místicas actúan para que las personas alcancen algún grado de explicación del acontecimiento, pero también les señalan que realmente no tienen control sobre determinadas situaciones de la vida: «pues más que nada, este, un accidente es algo que no se lo espera uno o algo que no... cómo le diré... algo que tenía que pasar» (10).

En los testimonios se aprecian falta de control y la adjudicación de los eventos lesivos a fuerzas externas a la conducta humana. Además, se habla del nombrado fatalismo colectivista (Blanco \& Díaz, 2007), una actitud sumisa, una resignación compartida por una comunidad o un grupo social.

\section{El niño y la niña}

\section{¿Víctima propiciatoria?}

Si bien no se puede afirmar que el adulto culpa plenamente a quien resulta víctima de una serie de circunstancias, sí llega a responsabilizar a los pequeños (tres años de 
edad) por algunos rasgos comportamentales que los condujeron a ser víctimas de lesiones. ¿Los cuidadores buscan cómo disminuir su responsabilidad, pese a que el menor es más bien víctima de las circunstancias?: «Pero pos ella también es bien terquecita y también aferrada a las cosas» (o1).

\section{¿El niño y la niña ya razonan?}

La psicología del aprendizaje ha dado a conocer que la presencia del adulto es, muchas veces, una especie de estímulo — condicionamiento operante- que detiene ciertos comportamientos exploratorios riesgosos de los pequeños; incluso la simple expresión verbal «no!» llega a ser efectiva. Sin embargo, dicha «obediencia» no significa la comprensión de las consecuencias, o sea, el entendimiento cognitivo de la causalidad (Piaget, 1995). El adulto confunde a menudo este tipo de aprendizajes o reacciones de los niños debido a que considera que obedece — «ya sabe»— porque ya tiene cierto grado de razonamiento. Por desgracia, lo anterior muchas veces redunda en una mayor confianza del adulto con respecto a su cuidado: «ella ya sabe que eso no se debe de agarrar ni para jugar ni para nada, pero ya está grande [cuatro años de edad] y ya capta más las cosas... Ella ya sabe lo que es peligro, lo que es veneno también, ella ya sabe todo eso» (o6).

La pedagogía conductista fracasa porque los niños y niñas como seres humanos están provistos de una esfera afectiva y de sentimientos que van más allá de los esquemas estímulo-respuesta.

\section{Discusión}

La exploración de la percepción de riesgo en la presentación de lesiones muestra importantes detalles de lo cotidiano, del cuidador, del riesgo y de los niños. Estos últimos tres elementos generales conforman las lesiones infantiles y advierten la necesidad de analizarlos e interrelacionarlos. El estudio de las lesiones a través de la narrativa permite entender cómo las personas enfrentan los retos y condiciones que impone la cotidianidad de su estilo de vida.

Los cuidadores percibieron las lesiones no intencionales como accidentes y las consideraron como parte del azar, hasta el punto de que luego del accidente predominó en ellos una actitud fatalista al estimar que no podían haber hecho nada para impedirlo. Así las cosas, la responsabilidad del accidente se atribuyó en mayor medida a un factor 
de control externo, aspecto con el cual también se aligeró la responsabilidad, en el sentido de disminuir el efecto emotivo del evento al otorgarle un sentido y una explicación (Oltedal et al., 2004; Rickard, 2014).

Se observó que aun cuando la percepción del riesgo existía, los cuidadores no aplicaron medidas de seguridad, confiados en que previamente no habían sucedido accidentes. Esto permite afirmar que lo que hemos denominado inexperiencia del daño influye de manera importante.

Similar a lo señalado en algunas investigaciones (Kouabenan, 2007), en el presente estudio las cuestiones culturales influyeron en la forma de percibir una situación de riesgo. Además, el aspecto cognitivo de los cuidadores implicó la adopción de una serie de actitudes, traducidas en acciones, que incidieron en la ocurrencia de las lesiones.

Al igual que en otras indagaciones (European Child Safety Alliance, 2001), se encontró que la seguridad de los niños parece no constituir una prioridad permanente en el hogar, sino que regularmente se otorgaba primacía a otras cuestiones, aspecto este que se traducía en una falta de atención a los riesgos, ya fuera por costumbre, rutinas o falta de recursos económicos para adecuaciones físicas y de prevención en el hogar. La exposición al riesgo se acompaña de escenarios de sobrecarga de cuidados, o de uso de productos peligrosos que no concilian la higiene con la seguridad, por lo que las políticas sanitarias tienen un área de oportunidad en este sentido.

Como se ha señalado (Okuda, Buriola, Teixeira, Da Silva, \& Félix, 2013; Sastre-Paz, Zoni, Esparza-Olcina, \& Del Cura, 2016), la mayoría de las lesiones sucedieron en el hogar. Un factor explicativo de ello se debe a que, mientras no hubo lesiones, los cuidadores asumieron a lo largo del tiempo una actitud de confianza y seguridad, pese a la existencia de medidas no del todo seguras y a riesgos latentes. Estas medidas pseudopreventivas tomadas por los cuidadores revelan el fenómeno señalado como pseudocerteza (Slovic, 200o); es decir, existió una manipulación en la creencia de que las medidas de control implementadas protegían, cuando en realidad no fue así. La experiencia de seguridad generada por la costumbre condicionó un aprendizaje significativo (Vygotsky, 1978) que se continuó reforzando por las rutinas diarias y su aspecto pragmático. Cuando las decisiones se toman de manera cotidiana en un área específica (como el hogar), con la creencia de que se controlan, se siente que hay menor riesgo (Deloitte Touche Tohmatsu, 2017). Las familias señalaron que nunca había habido una lesión en un largo tiempo, y en este sentido, la rutina impidió que se visualizara el riesgo real en el hogar. 
Los padres de familia identificaron el riesgo de una manera velada, y en ciertos casos responsabilizaron a los propios niños por algún rasgo de su comportamiento. Por otra parte, coincidimos con algunos investigadores (Okuda et al., 2013) en que se subestimó la capacidad de los niños y niñas para superar los obstáculos que los cuidadores habían implementado para limitar o inhibir los riesgos.

En varios casos, las lesiones se presentaron aun cuando un adulto estaba presente, pero se comprobó que los cuidadores efectuaban otras actividades en forma simultánea. Algunos de los padres argumentaron que realmente era imposible estar pendientes todo el tiempo (European Child Safety Alliance, 2001). Una condición elemental para disminuir los riesgos en el hogar es la comunicación, ya sea para informar acerca de las condiciones de este, o bien para poner al tanto de qué tipo de insumos riesgosos hay en la casa (Ferrer \& Klein, 2015).

Debido al orden de género prevaleciente, en la mayoría de los casos la madre resultó responsable del cuidado del menor y quien estuvo durante la hospitalización. Por su parte, el padre de familia, en los casos en que era el cuidador, condicionó su presencia en el hospital para el cuidado del menor.

Las lesiones tornan vulnerables a los menores y a la propia familia. La necesidad de hospitalización altera de manera significativa la vida cotidiana y la funcionalidad familiar.

Una de las limitaciones del estudio es que los resultados obtenidos se circunscriben culturalmente a la población estudiada, pero no hay fácil acceso a hospitales privados; además, las lesiones son más frecuentes en población de escasos recursos económicos. Sin embargo, los resultados establecen la inconveniencia de señalar los accidentes como consecuencia de una «cultura de la pobreza» (Lewis, 1986). El presente estudio se debe entender solamente como un acercamiento cualitativo a la comprensión de las lesiones en niños y niñas.

\section{Conclusiones}

La investigación en lesiones señala que un evento social depende, entre otros factores, de los roles sociales, actitudes, género, percepciones, ideología y nivel socioeconómico, además de aspectos importantes acerca de un antes y un después de las lesiones.

No es suficiente observar un riesgo para actuar; es necesario creer que puede pasar una lesión para generar un cambio de comportamiento. La mayoría de los sistemas de 
cuidado familiar llegan a tener desajustes ante imprevistos o circunstancias apremiantes, por lo que la organización del sistema de cuidados puede quebrantarse y este puede tornarse riesgoso, así sea por breves minutos.

La cultura y las creencias desempeñan un papel clave para «dejar pasar» una situación que, a simple vista, es riesgosa. Asimismo, la forma y las condiciones de vida generan rutinas que brindan una falsa sensación de seguridad en el hogar al subestimar el riesgo, lo que no hace sino mantenerlo latente para una lesión. La simultaneidad de acciones dificulta el cuidado. Resulta indispensable contar con políticas públicas sobre distintos aspectos de prevención que garanticen y faciliten la seguridad en el hogar.

\section{Referencias}

Atak, N., Karaoglu, L., Korkmaz, Y., \& Usubutun, S. (2010). A household survey: Unintentional injury frequency and related factors among children under five years in Malatya. Turk J Pediatr, 52(3), 285-293.

Bernard, H. R. (1988). Research methods in cultural anthropology. Newbury Park: Sage.

Blanco, A., \& Díaz, D. (2007). El rostro bifronte del fatalismo: fatalismo colectivista y fatalismo individualista. Psicothema, $19(4), 55^{2-558 . ~}$

Bruner, E. (1986). Ethnography as narrative. En W. V. Turner, \& E. M. Bruner (Eds.), The anthropology of experience (pp. 139-155). Chicago: University of Illinois Press.

Cassus-Becerra, S. L., Fuentes-Vásquez, C. G., \& Valdés-Valenzuela, C. G. (1998). Actitudes de autocuidado de embarazadas con respecto a alimentación, accidentes y salud oral de sus futuros hijos (pp. 143-157). Santiago de Chile: s.n.

Chardon, M. (2008). Representaciones sociales del cuidado: entre las prácticas y la noción de alteridad. Archivos Brasileiros de Psicología, 6o(2), 10-19.

Davis, R., \& Pless, B. (2001). BMJ bans «accidents». BMJ, 322, 1320-1321. https://doi.org/ 10.1136/bmj.322.7298.1320

Deloitte Touche Tohmatsu. (2017). From risk perception to safe behaviour. Recuperado de: http://www.deloitte.com.au/media/docs/au_Deloitte_from_risk_perception_to_safe_behaviour.pdf

De Souza Minayo, M. C. (1995). El desafío del conocimiento. Buenos Aires: Lugar Editorial. European Child Safety Alliance. (2001). Parent's perceptions of child safety. A 14 country study. Amsterdam: European Consumer Safety Association.

Ferrer, R., \& Klein, W. (2015). Risk perceptions and health behavior. Curr Opin Psychol, 5 , $85-89$. 
Foster, G. M. (1988). Las culturas tradicionales y los cambios técnicos. Ciudad de México: Fondo de Cultura Económica.

García, B., \& De Oliveira, O. (1998). Trabajo femenino y vida familiar en México. Ciudad de México: El Colegio de México.

Geertz, C. (1988). La interpretación de las culturas. Barcelona: Gedisa.

Gurgel, A. K. C., \& Monteiro, A. I. (2016). Prevención de accidentes domésticos para niños: susceptibilidad percibida por los cuidadores. Rev pesqui cuid fundam, 8(4), 5126-5135. https//doi.org/10.9789/2175-5361.2016.v8i4.5126-5135

Hogan, C., Weaver, N., Cioni, C., Hamilton, A., \& Thompson, S. (2018). Parental perceptions, risks, and incidence of pediatric unintentional injuries. J Emerg Nurs, 44(3), 267-273. https://doi.org/10.1016/j.jen.2017.07.017

Kouabenan, D. (2007). Incertitude, croyances, et management de la sécurité. Le travail humain, 3(70), 271-287. https://doi.org/10.3917/th.703.0271

Kouabenan, D. (2009). Role of beliefs in accident and risk analysis and prevention. Safety Science, 47, 767-776. https://doi.org/10.1016/j.ssci.2008.01.010

Leeberk, R., Anuradha, R., Kuryan, G., \& Anuratha, B. (2017). Perception of unintentional childhood injuries among mothers in rural South India. Indian Journal of Public Health, 61(3), 211-214. https://doi.org/10.4103/ijph.ijph_162_16

Lewis, Ó. (1986). Ensayos antropológicos. Ciudad de México: Grijalbo.

Lourenço, J., Furtado, B., \& Bonfim, C. (2008). Exogenic poisoning in children assisted in a pediatric emergency unit. Acta Paulista de Enfermagem, 21(2), 282-286. https://doi.org/ 10.1590/s0103-21002008000200008

Marques, J., Bautista, M., Teixeira, B., Fortes, M., \& Sales, L. (2012). Representaciones sociales de los accidentes con materiales cortopunzantes. Rev. Latino-Am. Enfermagem, 2o(6), 1-10.

Okuda, E., Buriola, A., Teixeira, J., Da Silva, T., \& Félix, M. (2013). Factores asociados a intoxicación infantil. Esc Anna Nery, 17(1), 31-37.

Oltedal, S., Moen, B., Klempe, H., \& Rundmo, T. (2004). Explaining risk perception: An evaluation of cultural theory. Norwegian: Rotunde.

Organización Panamericana de la Salud. (2004). Lesiones no intencionales. Legislación en América Latina sobre programas preventivos y responsabilidades. Colisión y atropello por vehículos, caídas, quemaduras y ahogamientos. Washington, D. C.: Organización Panamericana de la Salud.

Piaget, J. (1995). Seis estudios de psicología. Bogotá, D. C.: Labor.

Rickard, L. (2014). Perception of risk and the attribution of responsibility for accidents. Risk Analysis, 34(3), 514-528. https://doi.org/10.1111/risa.12118 
Safiétou, M., \& Kouabenan, D. (2013). Effects of the feeling of invulnerability and the feeling of control on motivation to participate in experience-based analysis, by type of risk. Accident Analysis and Prevention, 51, 310-317. https://doi.org/10.1016/j.aap. 2012.11.026

Sastre-Paz, M., Zoni, A., Esparza-Olcina, M., \& Del Cura, M. (2016). Prevalencia y factores asociados a lesiones no intencionales. Pediatría Atención Primaria, 18(71), 253-258. Recuperado de: http://www.redalyc.org/articulo.oa?id=3666496060o6 Schutz, A. (1974). El problema de la realidad social. Buenos Aires: Amorrortu. Slovic, P. (2000). The perception of risk. Londres: Earthscan Publications. Slovic, P. (2012). Percepción del riesgo generado por eventos extremos. Nueva York: Palisades. Slovic, P., Fischhoff, B., \& Lichtenstein, S. (2011). Accident probabilities and seat belt usage: a psychological perspective. En P. Slovic (Ed.), The perception of risk (pp. 73-79). Londres: Earthscan. https://doi.org/10.1016/0001-4575(78)90030-1

Tocabens, B. (2011). Definiciones acerca del riesgo y sus implicaciones. Revista Cubana de Higiene y Epidemiología, 49(3), 470-481. Recuperado de: http://www.redalyc.org/articulo.oa?id=223221363013

Turner, V. (1986). Dewey, Dilthey, and drama: an essay in the anthropology of experience. En W. V. Turner, \& E. M. Bruner (Eds.), The anthropology of experience (pp. 33-43). Chicago: University of Illinois Press.

Vasvàri, T. (2015). Risk, risk perception, risk management a review of the literature. Public Finance Quarterly, 6o(1), 29-48.

Vygotsky, L. (1978). Pensamiento y lenguaje. Madrid: Paidós.

Weber, R. (1990). Basic content analysis. Thousand Oaks: Sage.

Whitehead, E., \& Owens, D. (2012). Parental perceptions of unintentional injury risks to children. International Journal of Health Promotion and Education, 50(1), 20-27. https:// doi.org/10.1080/14635240.2012.661953

Zayas-Mujica, R., Cabrera-Cárdenas, U., \& Cayón, D. (2007). ¿Accidentes infantiles o lesiones no intencionales? Revista Cubana Pediatría, 79(1). Recuperado de: http://scielo.sld.cu/scielo.php?script=sci_arttext\&pid=So034-75312007000100009 